\title{
Examining the Myths of Connected and Autonomous Vehicles: Analysing the Pathway to a Driverless Mobility Paradigm
}

\author{
Alexandros Nikitas, Eric Tchouamou Njoya, Samir Dani \\ Department of Logistics, Operations, Hospitality and Marketing, Huddersfield Business School, \\ University of Huddersfield, Queensgate, Huddersfield, HD1 3DH, UK
}

\begin{abstract}
Connected and autonomous vehicles (CAVs) could become the most powerful mobility intervention in the history of human race; possibly greater than the conception of the wheel itself or the shift from horse-carriages to automobiles. Despite CAVs' likely traffic safety, economic, environmental, social inclusion and network performance benefits their full-scale implementation may not be as predictable, uncomplicated, acceptable and risk-free as it is often communicated by a large share of automotive industries, policy-makers and transport experts. Framing an 'unproven', 'disruptive' and 'life-changing' intervention, primarily based on its competitive advantages over today's conventional automobile technologies, may create misconceptions, overreaching expectations and room for errors that societies need to be cautious about. This article 'tests' eleven myths referring to an overly optimistic CAVs' development and adoption timeline. This approach highlights unresolved issues that need to be addressed before an inescapable CAV-based mobility paradigm transition takes place and provides relevant policy recommendations of how to achieve that.
\end{abstract}

Keywords: Connected and Autonomous Vehicles; Driverless and Self-driving Technologies; Artificial Intelligence and Mobility; Smart Urban Futures; Transport Policy and Planning.

\section{Introduction}

The full-scale introduction of connected and autonomous vehicles (CAVs) will be a monumental milestone in the transition to a machine-led transport paradigm that would eclipse what has been for over a century now a human activity, driving. From an automation perspective CAVs will be able to recognise the scene, plan the path, and control the motion without any human input (Kato et al, 2015). Simultaneously, from a connectivity perspective CAVs will interact with their 'drivers', other road vehicles, different travel modes including non-motorised transport, road infrastructure spanning from traffic signals to street lighting and a control centre responsible for optimising road space allocation and coordinating traffic. CAVs are projected to minimise human errors, imprecision and unpredictability.

CAVs therefore have, in theory at least, the potential to completely transform urban futures, with a revolution in ground transport, regulations permitting, that could dramatically change the landscape of cities around the world and have an enormous socio-economic, spatial and mobility impact (Alessandrini et al., 2015). These impacts may be far-reaching on several levels entailing changes to i) the demand and behaviour side, ii) the supply of mobility services and iii) network and facility operational performance (Mahmassani, 2016).

The current status of automation development is intriguing both industry- and policy-wise. The companies that have already engaged with the creation of this new breed of vehicles, with some of them being responsible for millions of autonomously driven miles and some coming outside the traditional automotive industry circles, according to Nikitas et al. (2017a) are: Audi, Baidu, BMW, Daimler, Delphi, Didi Chuxing, Ford, General Motors, Honda, Huawei, Hyundai, Jaguar Land 
Rover, Lyft, Magna, Mercedes-Bosch alliance, Microsoft, nuTonomy, PSA, Renault-Nissan alliance, Samsung, Tesla, Toyota, Uber, Volkswagen Group, Volvo, Waymo (Google's self-driving cars project), ZF and Zoox. This will be a business arena of significantly different dynamics than the existing one for conventional cars; Information and Communication Technology (ICT) design considerations will be the focal point and the key competitive market advantage of the different automotive competitors.

The spread of early piloting exercises of humanly supervised Autonomous Vehicles (AVs) and the interest on the one hand to embrace legislative frameworks regulating driverless mobilities and on the other to support research and development initiatives is constantly increasing. Early versions of AVs are already tested in California with humans inside them at all times for supervision purposes; it is expected that California, despite a deadly accident that slowed down the process (described in section 3.5), will be one of the first states to put in place a complete set of regulations for the inclusion of unsupervised driverless cars in its travel eco-system. Florida, the test-bed of Ford's driverless and cloud-based technologies, has also drafted legislation on AVs that is not particularly restrictive. Germany has amended its Road Traffic Act to create a regulatory framework for driverless machines allowing automakers and technology companies to test AVs on public roads with people behind the wheel at all times. China, the world's largest automotive market, is expected to adopt some of Germany's laws on AVs.

In Sweden, Volvo's Drive Me project with the blessing of the authorities, intended to put a fleet of $100 \mathrm{AVs}$ in the hands of regular drivers on the public roads of Gothenburg with the promise that they will not need to constantly supervise the driving activities, as a means of understanding how these cars impact the quality of life and the urban environment (Victor et al., 2017). However, the goal of testing out these vehicles has now been delayed until 2021 after Volvo, famed for its advanced vehicle safety, decided it cannot meet its original deadline. In another part of Europe, the UK Government is actively committed in building a world-leading CAV testing environment and becoming a prime destination for the development of CAVs providing annually a substantial amount of funds for research and development (UK Government, 2017). Legislation to promote safe use of driverless cars in the UK is to be developed by the Law Commission and be ready as early as 2021. Similarly, the New Zealand Transport Agency and the Australian Department for Infrastructure, Regional Development and Cities support manufacturers and developers wanting to test and improve AV technologies and researchers wanting to explore the impacts of driverless vehicles and make recommendations for CAVs implementation in their respective countries (Australian Government, 2017; New Zealand Transport Agency, 2018). The current state of industrial and policy-making development provides evidence that significant progress has been achieved so far but nonetheless a universal shift to a machine-led mobility paradigm is not imminent any time soon.

Thus, studies looking to develop a roadmap that could inform and guide the full-scale launch of driverless vehicles, by identifying some key opportunities and challenges that the cities might face because of them, are more than ever needed. There are many questions to be addressed in relation to this technology uptake and a need to prioritise them as Parkin et al. (2018) suggests. It is crucial that stakeholders realise that if these questions are not answered adequately CAVs' potential may remain untapped leading, as Papa and Ferreira (2018) rightly argued, to a mobility future less usercentric that is below current expectations.

Henceforth, the paper introduces, through a mixture of findings founded on an evidence-based literature review and the preliminary results of the authors' recent and on-going studies, some of the key opportunities and challenges that CAVs will pose on society. At its core part the paper examines and critically discusses one-by-one 11 of the most 'typical' myths (all of them misconceptions or popular notions based on unrealistic expectations) referring to the CAVs development timeline and eventual full-scale launch. These have been short-listed by a thorough academic and technical literature review and by the previous exploratory empirical work of the authors (i.e. Nikitas et al., 2017a; 2017b). This is followed by a section referring to predictions and 
policy recommendations that provides some lessons for policy-makers and technology developers and suppliers. Finally, a conclusion section highlights the key contributions of the present article. This paper refers to road-based interventions for transporting people and primarily to the vehicles that are projected to be the direct successors of today's conventional automobiles; so autonomous rail, air and waterborne systems and freight are beyond this study's immediate scope.

\section{Background: The opportunities and challenges of CAVs}

This section provides a background to the present study by introducing a balanced account of the potential opportunities and concerns typically associated with CAVs today. Some of these issues led to or are directly linked with the myths discussed in the core part of the paper.

Recent studies (e.g. Clark et al., 2016; Fraedrich et al., 2018; Milakis et al., 2017; Nikitas, 2015; Thomopoulos and Givoni, 2015) and authors' on-going empirical research work identified some of the key likely benefits that will be related with the introduction of CAVs in a wide scale. These are listed in Table 1:

Table 1 CAVs Potential Benefits to Society and Users

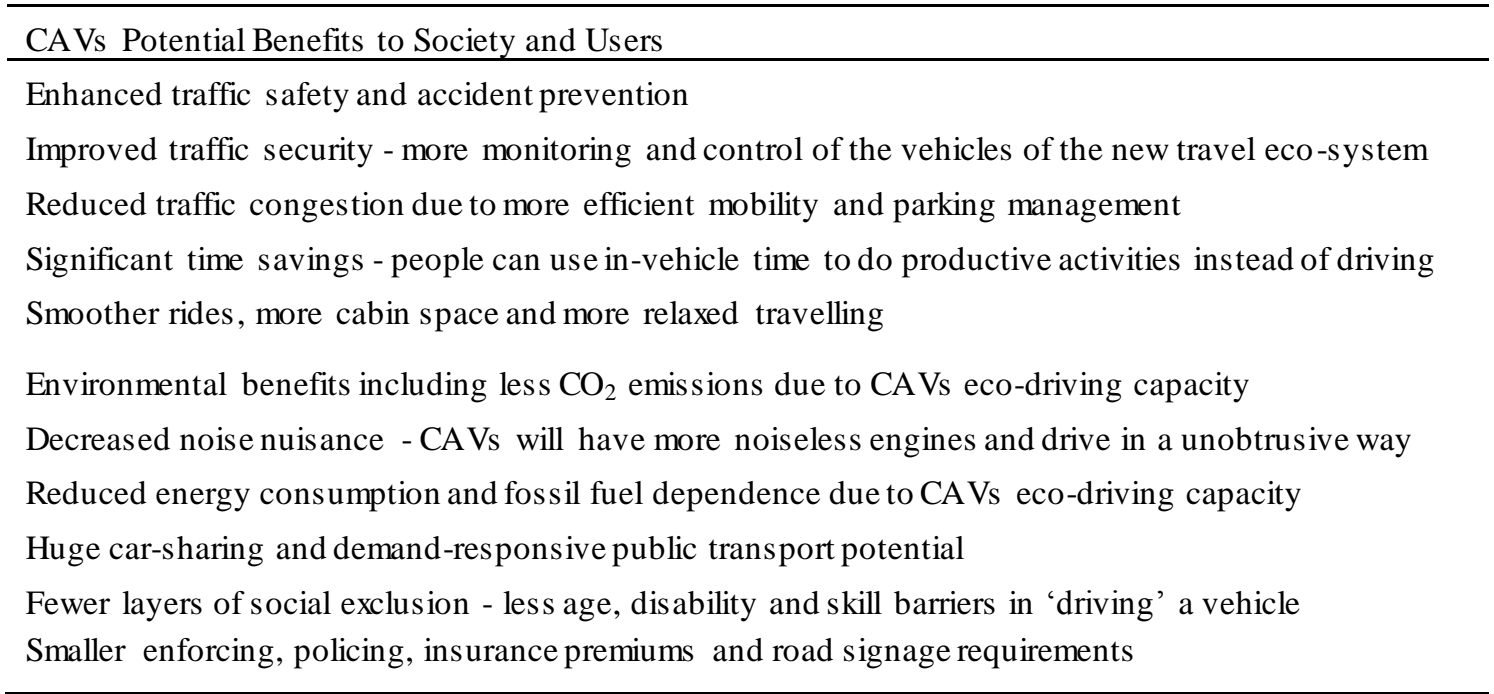

Despite these potential advantages and the fact that a priori acceptability of CAVs may be according to some tentative evidence (Payre et al, 2014) likely for many drivers today, transitioning from a human-led mobility paradigm to one dictated by Artificial Intelligence (A.I.) is a process of immense complexity and uncertainty. This is because, despite some initial encouraging technical results, the implementation of vehicle automation, may not be as straightforward, predictable, unproblematic or risk-free as it is often communicated by a significant share of the industry and policy stakeholders; there is a broad spectrum of challenges, social dilemmas and complex human factors issues that may arise from the introduction of such an 'untested' and 'powerful' intervention that should be resolved. Although generally not recognised as much as their potential benefits, CAVs could potentially be associated with numerous effects that could inconvenience urban societies and others that would directly affect their public acceptance.

Recent research (e.g. Cummings and Ryan, 2014a; Fagnant and Kockelman, 2015; Kaur and Rampersad, 2018; König and Neumayr, 2017) acknowledges some of these issues while the authors' past (e.g. Nikitas and Nikitas, 2015; Nikitas et al., 2017b) and on-going empirical research work identifies and puts together all the key problems with and arising from the implementation of CAVs; these include issues threatening uptake and potential adverse impacts generated by CAVs. These are presented in Table 2: 
Table 2 CAV-related Concerns for Society and Users

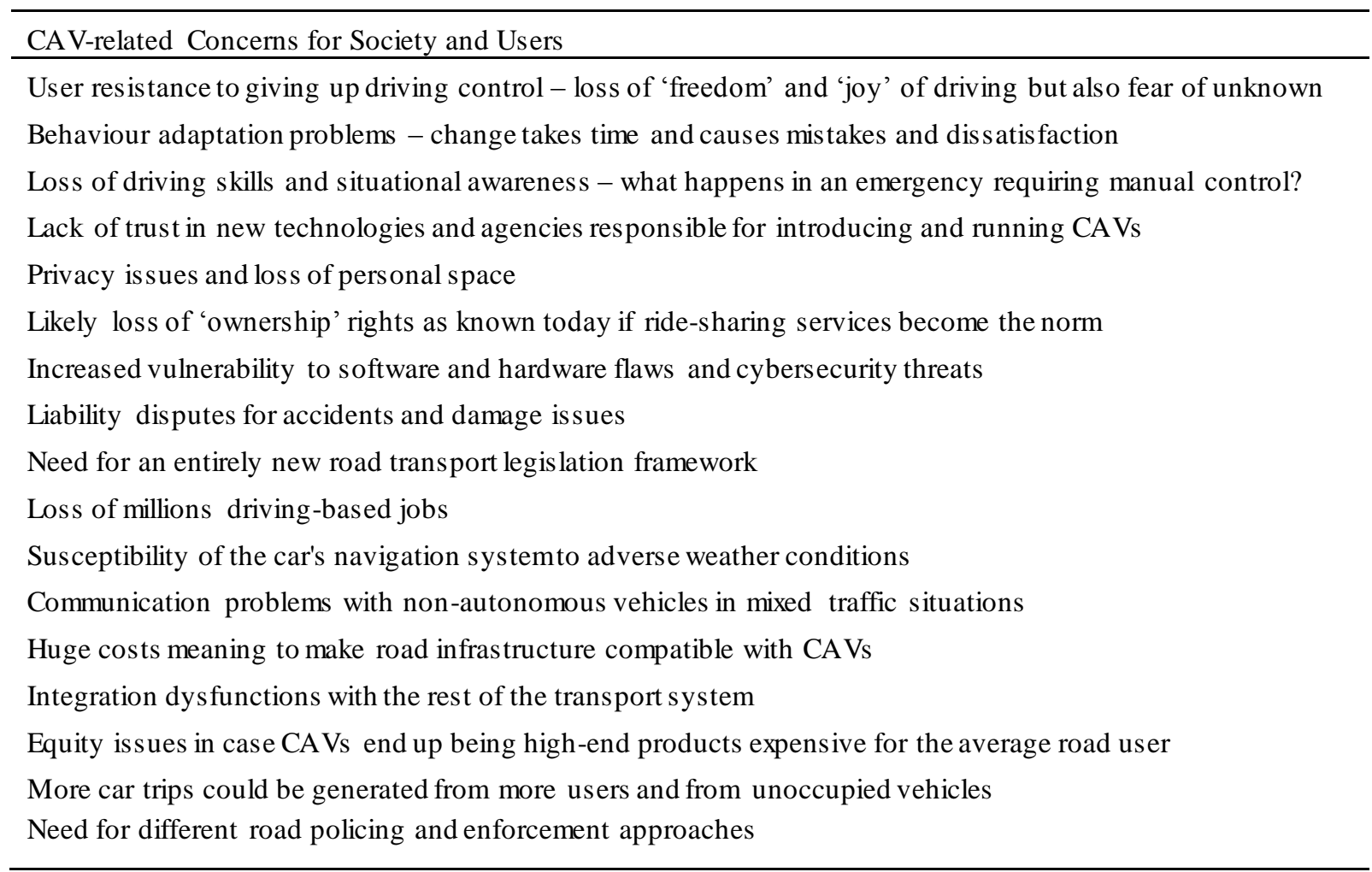

\section{CAV-related myths and their counterarguments}

This section will introduce and examine myths around the development and eventual implementation and uptake of CAVs that may not be accurate and could create misconceptions; all of them are overly optimistic about the degree of difficulty of the upcoming transition to a different mobility era. These myths have been introduced but not systematically analysed by the authors' previous empirical work (i.e. Nikitas et al., 2017a; 2017b).

\subsection{Myth one: Technology is already available}

Although many automotive companies are advertising the introduction of AVs by 2020 or sooner, while others claim that by 2021 they will be ready to mass produce Automated Driving Systems (ADS), all of them fail to clarify or choose not be specific about the type of automation features or operation modes in systems to be offered (Chan, 2017). This marketing approach probably 'admits' that despite optimism the era of CAVs is not arriving during the next handful of years; the first type of driverless-branded cars will probably be basic AVs that would be significantly inferior to fully developed CAVs in every technological aspect. Or as Litman (2017) suggests during their first decade AVs will represent a minority of vehicle sales because of significantly lower performance when compared with the subsequent generations of AVs.

The truth is that for now technology is still lacking. Despite significant progress and million miles driven by humanly supervised autonomous vehicle pilots in segmented or heavily controlled environments, many more breakthroughs are necessary for supporting a full-scale real-life launch of fully functional and connected self-driving vehicles. CAVs need to go beyond correctly detecting and identifying objects in typical transport scenarios; they need to be able to anticipate their behaviour even under the most complicated and unexpected circumstances (Nikitas et al., 2017a). Teaching the car how to respond to what can be described as 'the long tail of unlikely events' such as a plastic bag blowing across the motorway, a couch sitting in the middle of the road (Waldrop, 
2015) or unpredicted pedestrian of cyclist movement is of critical importance and is an accomplishment that is yet to be achieved in terms of technology.

According to Cummings and Ryan (2014b) the most difficult function that driverless technology needs to address is the one managing the interaction between the human driver and the robotic car. Human-machine interaction is still at initial stages for the case of driverless cars (Sheridan, 2016). Predicting and adjusting in real-time to human behaviour and 'communicating' effectively with a human passenger is a task that no machine has mastered yet, possibly because for now at least, humans and machines speak different languages. Humans are excellent in reasoning and planning in unstructured environments, while robots are very good in performing tasks repetitively and precisely (Musić and Hirche, 2017). Also the transition to CAVs will require currently available connectivity to give way to more advanced cooperative features that will facilitate safety and traffic information exchange among vehicles and infrastructure as Raposo et al. (2017) suggested.

\subsection{Myth two: Legislation changes are easy to design and deliver}

Despite a lot of interest to address it, legislation even in cases where there is strong support from local policy-makers to speed up usually lengthy consulting and legislative procedures, could be a barrier. Future road traffic regulations, liability allocation and enforcement strategies need to incorporate the use of CAVs meaning that these have to be re-thought and re-constructed. This is a radical switch for the current status quo and needs time, patience, political persistence and an understanding that changes need to be incremental and implemented on a trial and error basis. It should be realised that getting the legal framework right from day one for such an untested and 'game-changing' technology will not happen.

The legal landscape of the road transport automation including the product liability, cybersecurity, and intellectual property issues are of paramount concern (Sanitt et al., 2017). Defining the theme of responsibility adequately by developing meaningful distinctions and examining their ramifications is equally important (Liu, 2017). Among the legislative problems that still need to be solved before CAVs can autonomously participate in traffic is the one of making their behaviour conform to the traffic laws (Prakken, 2017); issues like exceptions, rule conflicts, open texture and vagueness, rule change, and the need for common-sense knowledge should be considered. In order to ensure legal compliance by self-driving cars, the regulator must supervise how programmers translate the norms of traffic into computer code, and must set standards of reliability for artificial fact-finding; crash algorithms in particular should not violate human dignity, should reflect the priorities of a legal order, and must not use personal characteristics such as race, gender, or age, to choose between potential victims of an accident (von Ungern-Sternberg, 2018).

\subsection{Myth three: Acceptability will be certain}

Although recent studies showed that, in theory, a priori acceptability of CAVs could be likely for many drivers today (Payre et al., 2014; Piao et al., 2016) the universal acceptance of CAVs, when these would not be a futuristic scenario anymore but an impeding reality, is not guaranteed or certain (Nikitas et al, 2017a). The public may need to overcome psychological challenges that stand in the way of widespread adoption (Shariff et al., 2017). Users might need to be convinced and trust is a process that takes time to develop when it comes to transport interventions (Nikitas et al., 2018). For instance, the acceptance of the much more market-ready and unambiguous electric vehicles is still marginal and their sales are very low (Biresselioglu et al., 2018) despite policymaking efforts to boost them; this is because people still do not trust enough these vehicles over conventional ones to pay the high premium for their purchase.

Attitudes towards fully automated driving (or higher levels of autonomy) range from 'excitement' to 'suspicion' with the breadth of feelings possibly relating to low levels of awareness 
or polarising attitudinal positions (Sun et al., 2017). One of the key issues is how much drivers will trust automated driving systems and how they calibrate their trust and reliance based on their experience; trust according to Dikmen and Burns (2017) may be correlated with several attitudinal and behavioural factors such as frequency of use, self-rated knowledge about these systems, and ease of learning. Daziano et al. (2017) found that the demand and willingness-to-pay for automation is split approximately evenly between high, modest and no demand, highlighting the importance of modelling flexible preferences for emerging vehicle technology.

Schoettle and Sivak (2014) suggested, based on 1533 responses, that many individuals express high levels of concern about: i) riding in self-driving vehicles; ii) security issues related to selfdriving vehicles; iii) self-driving vehicle not performing as well as actual drivers; iv) vehicles without driver controls; v) self-driving vehicles moving while unoccupied; and vi) self-driving commercial vehicles, buses, and taxis. Also, according Kyriakidis et al. (2015), a study based on an international survey of 5000 responses, people tend to see manual driving as the most enjoyable mode of driving and are concerned about software hacking/misuse, legal issues and safety.

Since acceptability is a key, and perhaps a pre-requisite, for the implementation of CAVs, car manufacturers and high technology firms should therefore invest on marketing campaigns for making customers receptive toward the benefits of autonomous driving with special attention, as Leicht (2018) suggests, to word-of-mouth and consumer-to-consumer marketing. Policy-makers facilitating this transition, on the other hand, will need to introduce education, training and in some cases consultation and engagement exercises that will help people appreciate the offerings of CAVs.

\subsection{Myth four: Implementation will be relatively straightforward}

The implementation of CAVs, even if public and therefore political acceptability is gained, will not be straightforward, predictable, unproblematic or without risks; there is a wide spectrum of issues that may arise from such an untested yet robust intervention that could prevent its flawless introduction in many cities. Experimental small-scale implementations in living labs cannot prepare urban societies for the breadth and depth of issues that will be associated with the full-scale launch of CAVs. There is a huge difference between building a few vehicles to run in reasonably benign conditions with professional safety drivers, and building a fleet of millions of vehicles that have to run in an unconstrained world (Koopman and Wagner, 2016).

According to Fagnant and Kockelman (2015) there are substantial barriers to implementation and mass-market penetration including unaffordable initial costs, inconsistencies and disparity in licensing and testing standards, liability issues, security and lack of privacy concerns and potential integration and interaction problems between CAVs and the other pieces of the transport network. However, because of CAVs' radical nature, which is directly associated with a high degree of uncertainty, there is a strong likelihood that entirely unforeseen, at present at least, challenges will arise with their introduction. If these barriers are not properly addressed in a way that will allow CAVs to be trusted by the majority of road users then a possibly 'incomplete' and 'rushed' implementation will create more problems than the ones it could be solving. Implementing CAVs need to happen when the technology, policy and legislation reach a level of maturity that will benefit the transport network and would not be a nuisance. The expansion of CAVs should be in that sense incremental starting from smaller scale controlled schemes; the more mature CAVs and their infrastructure become the wider their expansion should be. Low level automation should be allowed and carefully promoted but under the active supervision of human drivers.

\subsection{Myth five: Physical or cyber threats are smaller barriers than what people think}

One very important issue that can control the acceptability and reluctance narratives is how CAVs could get over the storm of a catastrophic event, like a fatal accident, that may highlight the prematurity of today's readiness for real-life testing. Over-reaction to accidents could be a 
roadblock to adoption (Shariff et al., 2017). An early example of that is the incident that took place in March 2018. A self-driving Uber car killed a woman in Arizona in the first fatal crash involving a pedestrian. This was an unfortunate event that created extremely negative publicity for the company and the autonomous vehicle technologies in general, pushing Uber, one of the premier industry players behind the drive to rush the transition to robotic cars, to re-configure its selfdriving aspirations and perhaps to a degree take a step back. This disaster will also make regions eager to serve as test-beds for CAVs to re-evaluate their rationale and be more cautious.

Another disaster scenario that could significantly reduce the acceptance of CAVs is one that refers to this technology's vulnerability to cyber threats and data sharing exploitation. As with all connected computing infrastructures, increasing the level of computational functionality and connectivity increases the exposure of potential vulnerabilities (Parkinson et al., 2017) and opportunities for data mismanagement. Unauthorised private data sharing, hacking and cyberterrorism are threats that could be easily associated with a less effective protection system for CAVs. Insufficient cybersecurity of CAVs can also expose a nation's critical infrastructure to cyber threats that can disrupt the delivery of critical services and have a detrimental impact on the entire society's wellbeing (Lim and Taeihagh, 2018). People may need to be convinced that CAVs provide a safe and secure environment that at the same time respects their privacy before they are to fully embrace them.

\subsection{Myth six: Ethics is a secondary issue comparing to other more technical considerations}

CAVs will need to operate responsibly and replicate or do better than the human decision-making process; as long as driverless vehicles are not safer than human drivers, it will be perhaps unethical to sell them (Sparrow and Howard, 2017). Nevertheless, even when this level of machine-led driving performance is achieved some decisions, as Lin (2016) suggests, will be more than just a mechanical application of traffic laws and plotting a safe path. CAVs are expected to crash occasionally, even when all sensors, vehicle control components, and algorithms function perfectly and if a human driver is unable to take control in time, a computer will be responsible for pre-crash behaviour (Goodall, 2014). Therefore if CAVs are going to drive on roads, it must be decided who is to be held responsible in case of traffic accidents (Hevelke and Nida-Rümelin, 2015) and what would be the computer's decision-making criteria for choosing from a set of crash trajectory alternatives; this involves not only legal questions, but more importantly, moral ones.

Ethics is a critical issue that underpins the introduction and usage of CAVs therefore. Even when it becomes possible to programme decision-making based on moral principles into machines, will self-interest or the public good prevail? CAVs will sometimes have to choose between two evils, such as running over pedestrians or sacrificing themselves and their passengers to save the pedestrians (Bonnefon et al., 2016) and there is not yet a clear consensus of what is the 'right' option. Establishing what kind of decision and behaviour is morally permitted, prohibited, or obligatory in such emergency situations is a hard philosophical problem (de Sio, 2017) that seeks an answer convincing enough to inspire trust and acceptability to CAVs. Actually, recent literature (i.e. Pugnetti and Schläpfer, 2018) reports that there is contradiction between studies about accident ethics conducted in different countries, potentially indicating the need to adapt both corporate communications and steering algorithms in different geographies. Ro and $\mathrm{Ha}$ (2019) provide empirical evidence via a survey study that if expectations that autonomous cars will make ethical decisions in emergencies increase, positive attitudes will be formed toward their adoption and use.

As a whole, this paper emphasizes the importance of the ethical implications that CAVs would introduce to the travel eco-systems of the future suggesting the need for creating a human-machine ethics paradigm, which currently does not exist. Allocating risks and responsibilities without a human driver's input is an issue with enormous moral dimensions, especially in cases where an autonomous car would not be able to avoid a collision path. 


\subsection{Myth seven: No driving, no problem}

Mental processes that are instrumental for human-led driving and depend on engagement and connection might become redundant for the passengers of CAVs. The passive human role when using a fully automated car in the future may not allow users to build an appropriate mental model of the situation that is essential for the recovery of a possible system failure (Breton and Bossé, 2003). This loss of the skills needed to perform the automated functions manually is called loss of situational awareness (Parasuraman et al., 2000) and is attributed to drivers being out-of-the-loop (Larsson, 2017; Strand et al., 2014). When abandoning a task for a while, that is associated with systematic practice as much as driving is, it is natural to lose the feel for it and underperform when there is a need to take over the control of the vehicle. More specifically, an automation conundrum exists in which as more autonomy is added to a system, and its reliability and robustness increase, human operators' situational awareness will decrease and it would be less likely that they will be able to take over manual control when needed (Endsley, 2017). Similarly Clark et al. (2017) identified through simulating automated scenarios that there is a link between lower levels of situational awareness and longer time to respond to a takeover event, while Louw et al. (2015) using desktop driver simulators suggested that drivers experiencing automation were slower to identify the potential collision scenario from when they had manual control.

Another issue with the introduction of CAVs resides in the fact that drivers can react in unexpected ways to the introduction of new systems, a phenomenon defined as 'behavioural adaptation' (Gouy et al., 2014). Carr (2015) highlights that future users may also suffer from disengagement and discontent. This is because, as Steg (2005) shows, the utility of car travel is not only dependent on its instrumental value; people do not only drive their car because it is necessary to do so, but also because they enjoy driving. If manual driving is taken from individuals that see car travel as a manifestation of joy or independence the user experience will never be the same for them; these particular commuters will be uninterested and uninspired by what CAVs will be offering and therefore might strongly oppose the concept creating resistance and potentially a threat to CAVs' universal uptake as the mainstream mobility paradigm of the future.

\subsection{Myth eight: The need for road infrastructure changes and investments is easily manageable}

Studies suggest that in countries which already have extensive road networks, it is hard to see segregation for autonomous vehicles being a viable proposition in all but a few extremely limited applications; e.g. for shuttle services running on private premises (Johnson, 2017). This however does not mean that the need for infrastructure investments would be a small one even in that scenario. CAVs because of their superior technologies and their need to be fully connected with each other and all the agents of a transport network will not be able to adequately function in the existing road transport landscape, because this is purely designed for conventional human-driven automobiles. CAVs will need a more tailored road transport environment that caters for their special requirements for connectivity, communication and coordination; so enhanced and in some cases new and smarter infrastructure would be necessary. This smarter road transport environment will include sensors, data capture capabilities, state-of-the-art CCTV and traffic signal systems, the ability to be responsive to changes in the environment, electrical charging provision, adaptable street lighting and more importantly the ability to 'talk' to different vehicles and road users.

In line with this, Gopalswamy and Rathinam (2018) suggested that infrastructure is a critical enabler for autonomy while Menouar et al. (2017) argued that there is still a significant need to automate other road and transportation components and not only the vehicles per se. Autonomy could require enhanced standards of road maintenance, to ensure that driverless vehicles are able to 'sense' the road environment accurately (Johnson, 2017). These infrastructure upgrades could be enormously expensive (Fagnant and Kockelman, 2015; Nikitas et al., 2017a) and form an initial barrier for the introduction of CAVs in a wide scale. Expensive transport infrastructure investments, due to limited availability of financial resources from governments, are always more difficult to 
decide, fund, design and implement, take time and need substantial and continuous political support.

\subsection{Myth nine: Mixed traffic situations present no threat}

If all the vehicles of a network are autonomous and connected to each other, a coordinated braking strategy can be devised to avoid both rear and front-end collisions improving traffic safety standards enormously (Patel et al., 2017). But this ideal scenario may not be easily implementable at least at the initial stages of a CAVs launch; replacing all the non-fully automated vehicles at once is unrealistic. The first generation of CAVs most likely will not run in isolation or within segregated road networks potentially because of the high cost involved as Johnson (2017) argued unless exclusive automated and/or regular vehicle lanes are adopted, which is a scenario involving huge road space re-organisation efforts. Mixed traffic situations, where CAVs share road space with highly automated, partially automated, and conventional human-driven vehicles could be the norm for an extended period of time, until a full conversion to driverless automobiles is achieved. However this mix could create many traffic safety and optimisation problems.

This is mainly because human-driven vehicles and CAVs have different driving logics (Talebpour and Mahmassani, 2016) and vehicle-to-vehicle connectivity and collaboration will not be the same. Unlike human piloted vehicles, CAVs have the capability to significantly reduce the headway between vehicles, potentially adding capacity without increasing the physical infrastructure (Wang et al., 2017) and eliminate human error, which is one of the most critical reasons for traffic accidents today. According to Arvin et al. (2018), mixed environments of humandriven and fully autonomous vehicles will still produce some safety benefits (e.g. traffic accident reduction) but significantly smaller than those of a solely CAV-based network.

\subsection{Myth ten: CAVs will be universally applied across the world in a few years}

There is a risk of creating a two- or even a three-speed world; continents, countries, regions and cities' progress in developing and introducing driverless technology may come at different rates and times. There will be a significant gap between the developed and the developing world's capacity to embrace this technology. This could potentially create imbalance, confusion and disharmony when transport's key objectives refer among others to integration, homogeneity, inclusion and equitable access. Also in line with what Johnson (2017) suggests, any system requiring extensive roadside communications technology, such as CAVs, could prove prohibitively expensive and unaffordable for some urban societies. This raises issues of international interoperability.

At the same time rural areas face completely different mobility challenges than urban areas; travel demand is significantly lower and more variable, public transport services may not be frequent, profitable or financially viable, road infrastructure is lacking and connectivity opportunities are fewer. CAVs design thus far is focusing on the environments and opportunities provided by cities and metropoles, where traffic levels are high and these expensive technologies make commercial sense. However for interoperability reasons at some point in the future extending some of the CAV-based services (or linking them at least) to rural areas should be also explored.

The timeline of a complete transition is also hard to predict but surely this change will not be realised in a matter of few years. Dokic et al. (2015) actually insisted that additional significant effort is required to create new concepts and test-systems for validation of complex AV systems in simulated environments before these can ever be applied in practice. Bansal and Kockelman (2017) suggested that, without a rise in most people's willingness to pay, or policies that promote or require technologies, or unusually rapid reductions in technology costs, it is unlikely that the U.S. will have a light-duty vehicle fleet with a technology mix that will be anywhere near homogeneous by the year 2045. And U.S. is actually one of the most transition-ready countries in terms of technology, financial capability and legislative flexibility in regards of adopting AVs (Bansal and 
Kockelman, 2018). Clark et al. (2016) actually expects that automation will first be widely applied to closed urban rail systems rather than to personal road-based transport something that early applications like London's Docklands Light Railway and Heathrow's self-driving pods do confirm.

\subsection{Myth eleven: Business models for CAVs have been established}

The automotive industry has reached the end of a prolonged period of technological monoculture that allowed and necessitated the kind of monolithic industrial structure, business models and operational practices currently in evidence (Wells, 2010). CAVs will change the way the automotive industry functions and this will make necessary the establishment and adoption of a different business model approach. Car-makers will need to compete and collaborate with their new competitors, actors outside the traditional vehicle manufacturers, such as Google, Tesla, Uber, and impose new business models that fit the challenge of the driverless vehicle (Attias, 2017). These business models and their respective policy paths should be flexible and resilient to uncertainties as suggested by Lyons (2016), while their viability should be primarily evaluated based on the competitiveness of their cost structures (Bösch et al., 2018).

One of the more promising operational opportunities is the concept of shared fully-automated vehicles (SAVs) which will transform the notion of travel from one that is largely by privately held personal vehicles to fleet services by driverless, demand-responsive vehicles, shared (or for hire) across a mix of users (Fagnant and Kockelman, 2018). As attitudes toward owning versus leasing, renting and sharing transport vehicles are changing (Tinnilä and Kallio, 2015) this business model could be soon more acceptable especially from younger people. Stocker and Shaheen (2018) actually make the case that AVs, if shared, have the potential to blur the lines between public and private transportation services altogether. There are advocates according to Litman (2017) suggesting that private vehicles will be eventually entirely displaced by automated taxi-like services. A private ownership-based automation paradigm may be problematic because of the high cost of the vehicles and the need for a higher level of coordination that isolated ownership could not offer. On the other hand, the impersonal nature of SAVs suggests that for some individuals, shared services may not be able to satisfy symbolic-effective motives, such as the use of the car as symbol of social status and self-expression as well as feelings of autonomy, freedom and flexibility, to the same degree as a private autonomous car will be able to do (Krueger et al., 2016). Thus, private vehicle ownership will likely endure the rise of such new technologies (Bösch et al., 2018) but for the first time may be out-shadowed by ride-sharing services.

Taking this shared use mobility concept a step further it can be hypothesised that SAVs will be an integral part of a holistic transport provision regime known as Mobility-as-a-Service (MaaS). $\mathrm{MaaS}$ is a mechanism replacing privately owned transport with personalised mobility packages that give access to multiple travel modes, smart ticketing and real-time information on an as-needed basis via powerful digital platforms. A MaaS-based mobility paradigm will provide opportunities according to Hensher (2017) to improve the customer experience provided by automated public transport that would need to complement car-sharing schemes. Having viable automated public transport services will be the only way to genuinely achieve in providing a sustainable transport network because of its ability to carry a bigger number of road users that according to Villagra et al. (2012) is a key for a more efficient, traffic-free and less-polluting urban mobility.

\section{$4 \quad$ Predictions and policy recommendations}

Autonomy in general, and motion autonomy in particular, has been a longstanding issue in robotics (Benenson, 2008). Empowering robotic technology to take control of the car, making calculated decisions and interrelating in real-time with the road traffic environment to heights unprecedented for humans, is a decisive step towards transitioning to a new machine-led transport paradigm. Introducing A.I. to vehicle technology will arguably be a monumental achievement in the history of road transport, revolutionising mobility for ever and shaping the future of societies; but for now 
CAVs are still more of an enigma than a definitive answer to transport problems. This is because driverless technology is still in its infancy stage; there is a considerable road to travel, according to Bagloee et al. (2016), before maturity, implementation, and mass-market release are achieved. This road might be longer and more uphill than many stakeholders anticipate; changes of this monumental magnitude carry a high degree of uncertainty, complexity and difficulty. Research and development efforts should therefore, in line with what Russel et al. (2018) suggeted, investigate in a systematic way how to maximise the potential benefits of CAVs while lessening their potential adverse impacts.

This study's intention is to help informing the society and those responsible for putting together this mobility paradigm shift, like policy-makers and industrial stakeholders, about the magnitude and diversity of the steps underpinning a full-scale launch of CAVs. The eleven important lessons that derived from the analysis of the myths outlined herein are summarized in Figure 1.

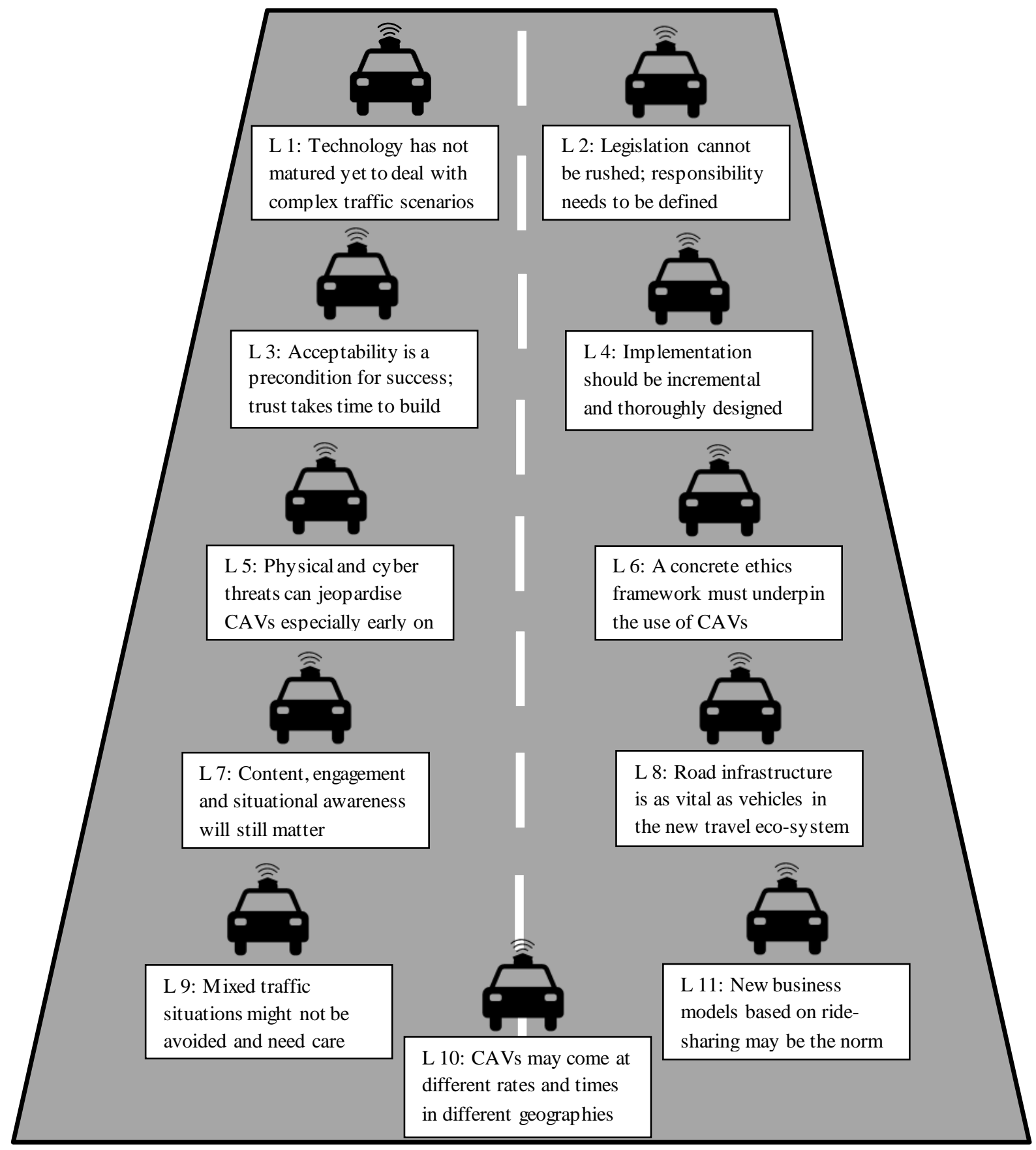

Figure 1: Lessons learnt by the examination of the 11 myths referring to CAVs 
The present work suggests that the shift to CAVs will require patience, political perseverance and flexibility coupled with continuous investment on technology, infrastructure, research and development. Shifting to an autonomous, connected and digitised mobility paradigm could be more complicated and difficult than many predictions suggest. Numerous trials will go wrong before the agents responsible for making CAVs mainstream get everything right. Thus, several piloting exercises in closely controlled environments will be necessary before a full-scale introduction is attempted so that problems will be timely identified and treated when still in an embryonic stage.

There will also be some failures, traffic accidents, cybersecurity breaches and even larger-scale catastrophes and fiascoes that could potentially create bad publicity, extra layers of public and political resistance and development stagnation. Strategic design, incremental changes, strong branding, marketing and communication schemes and being able to manage and respond to disappointments could protect CAVs early reputation and inspire road user trust. Different stakeholders, from automotive to computational industries and from international policy-makers to national, regional and city ones, need to collaborate effectively and have a homogeneous vision and a universal consensus about how they need to take every single step of this lengthy road and build trust. Trust as Pettersson and Karlsson (2015) conclude is a key issue for the successful introduction of CAVs.

It should be clear that CAVs are not a transport panacea; this advanced technology cannot solve all the mobility problems on its own. Instead CAVs constitute a significant new piece of a far more complex and multi-dimensional mobility puzzle. Similarly to what was concluded in Sochor and Nikitas (2016) 'technology is only one of the several tools in the toolbox of mobility'. Efforts should be directed beyond enhancing technology development per se; investors need to focus with similar tenacity to supportive instruments and institutions like traffic regulations, mobility education, marketing campaigns that enhance acceptability and adoption processes. Other, even wider, future mobility mechanisms like MaaS should incorporate CAVs effectively while travel demand management measures like road pricing and tollways will still be necessary. Public transport should remain a crucial part of the new automated travel eco-system; mass transit services based on buses and rail will always be a prerequisite for effectively addressing traffic congestion.

Unlimited automation of all technical functions will likely prove anathema to the fundamental quality of human life (Hancock, 2014) so there needs to be a balance in the cooperation, coordination and potential conflict between humans and machines. Human-machine interactions need to be effective for CAVs to thrive; creating disengaged passengers that would not be able to take over on an emergency situation is not the way forward. From an acceptability point of view early adopters need to be convinced promptly in the process of 'shifting' so that they will effectually propagate the message of 'embracing CAVs' (Nikitas and Nikitas, 2015). At the same time 'motives' or 'replacements' should be provided to car enthusiasts that view manual driving as an irreplaceable 'freedom' or 'joy'; this could include special circuits or automated-free areas.

The formation of a mixed traffic situation, where vehicles equipped with automated systems taking over the lateral and longitudinal control of the vehicle will interact with unequipped vehicles that are not fitted with such automated systems, may be another likely future scenario that could potentially create problems (Gouy et al., 2014). This inescapable co-existence of different driving technologies could be critical for the acceptance and uptake of CAVs. Early malfunctions need to be avoided in this pivotal stage of transition, and when segregation measures are possible or financially viable, these should be embraced.

Cultural and socio-economic issues could also play a role in how quick and efficient this transition process would be to different urban societies across the globe; the target is to increase the potential for interoperability and reduce isolation. Potential distributional impacts should be also closely monitored and controlled so that all road users enjoy the benefits of CAVs; ideally there should not be any winners and losers in terms of access to these vehicles. CAVs should be an inclusive and affordable technology and not one that creates new layers of transport-related social exclusion. 
Establishing an automated mobility paradigm where A.I. will be able to erase human error from the driving equation is an unprecedented challenge; one that has the potential to substantially improve the standard of transport offered. Despite a wide spectrum of potential traffic safety, socioeconomic, environmental, accessibility and network optimisation benefits though, that make CAVs an inescapable future direction for mobility provision, their full-scale implementation, may not be an easy step forward for the automotive industry, the policy-makers and the general public. Overcelebrating an 'unproven', 'novel' and 'transformative' intervention, primarily based on its theoretical competitive advantages over manually controlled automobile technologies, may create misunderstandings, deceiving expectations, an urge to rush an untimely implementation and room for errors and failures that could be costly to urban societies. The present article attempts to provide a critical examination of some typical misconceptions referring to CAVs' development and adoption readiness.

It ultimately makes the case that transitioning to a new machine-led transport era is not a straightforward and one-dimensional technology-centric process but a complicated multi-layer paradigm shift that needs: a lot more time and patience; huge investments in vehicle, road and wireless infrastructure technologies; collaboration between the different stakeholders responsible for it; strategic planning and incremental scheme expansion; new complementing legislative, moral and educational frameworks; fitting business models, based primarily on sharing options, that would create market penetration; activities building trust and acceptance; preparedness to manage failures or even catastrophes; and numerous testing and piloting schemes. If these preconditions for success are addressed in a timely and systematic way then CAVs will be an intervention that will significantly improve the travel eco-system of tomorrow. Future work should include empirical research that will examine in detail every single myth outlined herein as a means of improving and developing further the lessons learnt from this paper.

\section{References}

1. Alessandrini, A., Campagna, A., Delle Site, P., Filippi, F. and Persia, L. (2015). Automated vehicles and the rethinking of mobility and cities. Transportation Research Procedia, SIDT Scientific Seminar 2013, 5, 145-160.

2. Arvin, R., Kamrani, M., Khattak, A. J. and Rios-Torres, J. (2018). Safety Impacts of Automated Vehicles in Mixed Traffic. Transportation Research Board $97^{\text {th }}$ Annual Meeting, January, Washington, USA.

3. Attias, D. (2017). The Autonomous Car, A Disruptive Business Model?. In The Automobile Revolution, 99-113. Springer, Cham.

4. Australian Government (2017). Automated Vehicles in Australia. Available online: https://infrastructure.gov.au/transport/automatedvehicles/index.aspx (accessed 4 June 2018).

5. Bagloee, S. A., Tavana, M., Asadi, M. and Oliver, T. (2016). Autonomous vehicles: challenges, opportunities, and future implications for transportation policies. Journal of Modern Transportation, 24(4), 284-303.

6. Bansal, P. and Kockelman, K. M. (2018). Are we ready to embrace connected and self-driving vehicles? A case study of Texans. Transportation, 45(2), 641-675.

7. Bansal, P. and Kockelman, K. M. (2017). Forecasting Americans' long-term adoption of connected and autonomous vehicle technologies. Transportation Research Part A: Policy and Practice, 95, 49-63. 
8. Benenson, R., Petti, S., Fraichard, T. and Parent, M. (2008). Towards urban driverless vehicles. International Journal of Vehicle Autonomous Systems, 6(1-2), 4-23.

9. Biresselioglu, M. E., Kaplan, M. D. and Yilmaz, B. K. (2018). Electric mobility in Europe: A comprehensive review of motivators and barriers in decision making processes. Transportation Research Part A: Policy and Practice, 109, 1-13.

10. Bonnefon, J. F., Shariff, A. and Rahwan, I. (2016). The social dilemma of autonomous vehicles. Science, 352, 1573-1576.

11. Breton, R. and Bossé, É. (2003). The Cognitive Costs and Benefits of Automation, Technical Report, Defence Research and Development Canada: Ottawa, ON, Canada, 1-12.

12. Bösch, P. M., Becker, F., Becker, H. and Axhausen, K. W. (2018). Cost-based analysis of autonomous mobility services. Transport Policy, 64, 76-91.

13. Carr, N. (2015). The Glass Cage: Where Automation Is Taking Us, 1st ed.; W.W. Norton \& Company Inc.: New York, NY, USA.

14. Chan, C. Y. (2017). Advancements, prospects, and impacts of automated driving systems. International Journal of Transportation Science and Technology, 6(3), 208-216.

15. Clark, H., McLaughlin, A. C. and Feng, J. (2017). Situational awareness and time to takeover: exploring an alternative method to measure engagement with high-level automation. In Proceedings of the Human Factors and Ergonomics Society Annual Meeting, 61(1), 14521456. Sage CA: Los Angeles, CA: SAGE Publications.

16. Clark, B., Parkhurst, G. and Ricci, M. (2016). Understanding the Socioeconomic Adoption Scenarios for Autonomous Vehicles: A Literature Review. University of the West of England Report, Bristol, UK.

17. Cummings, M. L. and Ryan, J. C. (2014a). Shared Authority Concerns in Automated Driving Applications. Humans and Automation Laboratory Report, Massachusetts Institute of Technology, USA.

18. Cummings, M. L. and Ryan, J. C. (2014b). Who is in charge? The promises and pitfalls of driverless cars. TR News, 292, 25-30.

19. Daziano, R. A., Sarrias, M. and Leard, B. (2017). Are consumers willing to pay to let cars drive for them? Analyzing response to autonomous vehicles. Transportation Research Part C: Emerging Technologies, 78, 150-164.

20. de Sio, F. S. (2017). Killing by autonomous vehicles and the legal doctrine of necessity. Ethical Theory and Moral Practice, 20(2), 411-429.

21. Dikmen, M. and Burns, C. (2017). Trust in autonomous vehicles: The case of Tesla Autopilot and Summon. In Systems, Man, and Cybernetics (SMC), 2017 IEEE International Conference on, 1093-1098. IEEE.

22. Dokic, J., Müller, B. and Meyer, G. (2015). European Roadmap Smart Systems for Automated Driving. European Technology Platform on Smart Systems Integration, Berlin, Germany.

23. Endsley, M. R. (2017). From here to autonomy: lessons learned from human-automation research. Human Factors, 59(1), 5-27.

24. Fagnant, D. J. and Kockelman, K. M. (2018). Dynamic ride-sharing and fleet sizing for a system of shared autonomous vehicles in Austin, Texas. Transportation, 45(1), 143-158.

25. Fagnant, D. J. and Kockelman, K. (2015). Preparing a nation for autonomous vehicles: opportunities, barriers and policy recommendations. Transportation Research Part A: Policy and Practice, 77, 167-181. 
26. Fraedrich, E., Heinrichs, D., Bahamonde Birke, F. J. and Cyganski, R. (2018). Autonomous driving, the built environment and policy implications. Transportation Research Part A: Policy and Practice, In Press.

27. Goodall, N. (2014). Ethical decision making during automated vehicle crashes. Transportation Research Record: Journal of the Transportation Research Board, 2424, 58-65.

28. Gopalswamy, S. and Rathinam, S. (2018). Infrastructure Enabled Autonomy: A Distributed Intelligence Architecture for Autonomous Vehicles. arXiv preprint arXiv:1802.04112.

29. Gouy, M., Wiedemann, K., Stevens, A., Brunett, G. and Reed, N. (2014). Driving next to automated vehicle platoons: How do short time headways influence non-platoon drivers' longitudinal control? Transportation Research Part F: Traffic Psychology and Behaviour, 27(B), 264-273.

30. Hancock, P. A. (2014). Automation: how much is too much?. Ergonomics, 57(3), 449-454.

31. Hensher, D. A. (2017). Future bus transport contracts under a mobility as a service (MaaS) regime in the digital age: Are they likely to change?. Transportation Research Part A: Policy and Practice, 98, 86-96.

32. Hevelke, A. and Nida-Rümelin, J. (2015). Responsibility for crashes of autonomous vehicles: an ethical analysis. Science and Engineering Ethics, 21(3), 619-630.

33. Johnson, C. (2017). Readiness of the Road Network for Connected and Autonomous Vehicles. Royal Automobile Club Foundation, London, UK.

34. Kato, S., Takeuchi, E., Ishiguro, Y., Ninomiya, Y., Takeda, K., and Hamada, T. (2015). An open approach to autonomous vehicles. IEEE Micro, 35(6), 60-68.

35. Kaur, K. and Rampersad, G. (2018). Trust in driverless cars: Investigating key factors influencing the adoption of driverless cars. Journal of Engineering and Technology Management, 48, 87-96.

36. Koopman, P. and Wagner, M. (2016). Challenges in autonomous vehicle testing and validation. SAE International Journal of Transportation Safety, 4(1), 15-24.

37. Krueger, R., Rashidi, T. H., and Rose, J. M. (2016). Preferences for shared autonomous vehicles. Transportation Research Part C: Emerging Technologies, 69, 343-355.

38. Kyriakidis, M., Happee, R. and de Winter, J. C. (2015). Public opinion on automated driving: Results of an international questionnaire among 5000 respondents. Transportation Research Part F: Traffic Psychology and Behaviour, 32, 127-140.

39. König, M. and Neumayr, L. (2017). Users' resistance towards radical innovations: The case of the self-driving car. Transportation Research Part F: Traffic Psychology and Behaviour, 44, 42-52.

40. Larsson, A. (2017). A Countdown to Manual Driving: How Do Drivers Get "Back-in-theLoop"?. In Advances in Human Aspects of Transportation, 463-471. Springer, Cham.

41. Leicht, T., Chtourou, A. and Youssef, K. B. (2018). Consumer innovativeness and intentioned autonomous car adoption. The Journal of High Technology Management Research, 29(1), 111.

42. Lim, H. S. M. and Taeihagh, A. (2018). Autonomous Vehicles for smart and sustainable cities: an in-depth exploration of privacy and cybersecurity implication. Energies, 11(5), 1062.

43. Lin, P. (2016). Why Ethics Matters for Autonomous Cars. In Autonomous Driving; Maurer, M., Gerdes J., Lenz B., Winner H., Eds., 69-85, Springer: Berlin/Heidelberg, Germany.

44. Litman, T. (2017). Autonomous Vehicle Implementation Predictions. Victoria Transport Policy Institute, Victoria, Canada. 
45. Liu, H. Y. (2017). Irresponsibilities, inequalities and injustice for autonomous vehicles. Ethics and Information Technology, 19(3), 193-207.

46. Louw, T., Merat, N. and Jamson, H. (2015). Engaging with highly automated driving: to be or not to be in the loop? Proceedings of the Eighth International Driving Symposium on Human Factors in Driver Assessment, Training and Vehicle Design, June, 2015, Salt Lake City, Utah. Iowa City, IA: Public Policy Center, University of Iowa, 190-196.

47. Lyons, G. (2016). Transport analysis in an uncertain world. Transport Reviews, 36(5), 553557.

48. Mahmassani, H. S. (2016). 50th anniversary invited article-autonomous vehicles and connected vehicle systems: Flow and operations considerations. Transportation Science, 50(4), 1140-1162.

49. Menouar, H., Guvenc, I., Akkaya, K., Uluagac, A. S., Kadri, A. and Tuncer, A. (2017). UAVenabled intelligent transportation systems for the smart city: Applications and challenges. IEEE Communications Magazine, 55(3), 22-28.

50. Milakis, D., Van Arem, B. and Van Wee, B. (2017). Policy and society related implications of automated driving: a review of literature and directions for future research. Journal of Intelligent Transportation Systems, 21(4), 324-348.

51. Musić, S. and Hirche, S. (2017). Control sharing in human-robot team interaction. Annual Reviews in Control, 44, 342-354.

52. New Zealand Transport Agency (2018). Testing Autonomous Vehicles in New Zealand. Available online: https://www.nzta.govt.nz/vehicles/vehicle-types/automated-and-autonomousvehicles/testing-autonomous-vehicles-in-new-zealand/ (accessed 10 May 2018).

53. Nikitas A. (2015). Automated Cars: A critical review of the potential advantages and disadvantages of driverless technologies. First International Workshop on Smart Urban Mobility, November, Edinburgh Napier University, Edinburgh, UK.

54. Nikitas, A., Avineri, E. and Parkhurst, G. (2018). Understanding the public acceptability of road pricing and the roles of older age, social norms, pro-social values and trust for urban policy-making: The case of Bristol. Cities, 79, 78-91.

55. Nikitas A., Kougias I., Alyavina E. and Tchouamou Njoya E. (2017a). How can Autonomous and Connected Vehicles, Electromobility, BRT, Hyperloop, Shared Use Mobility and Mobility-As-A-Service shape transport futures for the context of smart cities?. Urban Science, 1 (4).

56. Nikitas A. and Nikitas G. (2015). Social dilemmas in vehicle automation. The 2015 Royal Geographical Society (with IBG) Annual International Conference, Special Transport Geography Research Group session in The Spaces of Road Transport Automation, September, Exeter, UK.

57. Nikitas A., Njoya T. E. and Dani S. (2017b). Examining the myths around autonomous cars: what does the future hold? 4th Smart Urban Policy Futures, July, University of Greenwich, London, UK.

58. Papa, E. and Ferreira, A. (2018). Sustainable accessibility and the implementation of automated vehicles: Identifying critical decisions. Urban Science, 2(1), 5.

59. Parasuraman, R., Sheridan, T. and Wickens, C. (2000). A model for types and levels of human interaction with automation. IEEE Transactions on Systems, Man, and Cybernetics Part A: Systems and Humans, 30, 286-297. 
60. Parkin, J., Clark, B., Clayton, W., Ricci, M., and Parkhurst, G. (2018). Autonomous vehicle interactions in the urban street environment: A research agenda. Proceedings of the Institution of Civil Engineers: Municipal Engineer, 171(1), 15-25.

61. Parkinson, S., Ward, P., Wilson, K. and Miller, J. (2017). Cyber threats facing autonomous and connected vehicles: future challenges. IEEE Transactions on Intelligent Transportation Systems, 18(11), 2898-2915.

62. Patel, R. H., Härri, J. and Bonnet, C. (2017). Braking strategy for an autonomous vehicle in a mixed traffic scenario. In Proceedings of the 3rd International Conference on Vehicle Technology and Intelligent Transport Systems, VEHITS 2017, April, Porto, Portugal.

63. Payre, W., Cestac, J. and Delhomme, P. (2014). Intention to use a fully automated car: Attitudes and a priori acceptability. Transportation Research Part F: Traffic Psychology and Behaviour, 27(B), 252-263.

64. Pettersson, I. and Karlsson, I. M. (2015). Setting the stage for autonomous cars: a pilot study of future autonomous driving experiences. IET Intelligent Transport Systems, 9(7), 694-701.

65. Piao, J., McDonald, M., Hounsell, N., Graindorge, M., Graindorge, T. and Malhene, N. (2016). Public views towards implementation of automated vehicles in urban areas. Transportation Research Procedia, 14, 2168-2177.

66. Prakken, H. (2017). On the problem of making autonomous vehicles conform to traffic law. Artificial Intelligence and Law, 25(3), 341-363.

67. Pugnetti, C. and Schläpfer, R. (2018). Customer preferences and implicit tradeoffs in accident scenarios for self-driving vehicle algorithms. Journal of Risk Financial Management, 11 (2), 28.

68. Raposo, M. A., Ciuffo, B., Makridis, M. and Thiel, C. (2017). From connected vehicles to a connected, coordinated and automated road transport (C 2 ART) system. In Models and Technologies for Intelligent Transportation Systems (MT-ITS), 2017 5th IEEE International Conference, 7-12, IEEE.

69. Ro, Y. and Ha, Y. (2019). A factor analysis of consumer expectations for autonomous cars. Journal of Computer Information Systems, 59(1), 52-60.

70. Russell, S., Dewey, D. and Tegmark, M. (2015). Research priorities for robust and beneficial artificial intelligence. AI Magazine, 36(4), 105-114.

71. Sanitt, A., Evans, M., Saddar, S., Evans, H. and Hidaka, S. (2017). Autonomous Vehicles: The Legal Landscape Of DSRC In The United Kingdom In Autonomous Vehicles: The Legal Landscape Of Dedicated Short Range Communication In The US, UK And Germany. Report, Norton Rose Fulbright.

72. Schoettle, B. and Sivak, M. (2014). A survey of public opinion about autonomous and selfdriving vehicles in the U.S., the U.K., and Australia. University of Michigan, Michigan, USA. Available online: http//deepblue.lib.umich.edu/bitstream/handle/2027.42/108384/103024.pdf. (accessed 10 June 2018)

73. Shariff, A., Bonnefon, J. F. and Rahwan, I. (2017). Psychological roadblocks to the adoption of self-driving vehicles. Nature Human Behaviour, 1(10), 694-696.

74. Sheridan, T. B. (2016). Human-robot interaction: status and challenges. Human Factors, 58(4), 525-532.

75. Sochor, J. and Nikitas, A. (2016). Vulnerable users' perceptions of transport technologies. Proceedings of the Institution of Civil Engineers: Urban Design and Planning, 169(3), 154162. 
76. Sparrow, R. and Howard, M. (2017). When human beings are like drunk robots: Driverless vehicles, ethics, and the future of transport. Transportation Research Part C: Emerging Technologies, 80, 206-215.

77. Steg, L. (2005). Car use: lust and must. Instrumental, symbolic and affective motives for car use. Transportation Research Part A: Policy and Practice, 39(2-3), 147-162.

78. Stocker, A. and Shaheen, S. (2018). Shared Automated Mobility: Early Exploration and Potential Impacts. In Road Vehicle Automation 4, 125-139. Springer, Cham.

79. Strand, N., Nilsson, J., Karlsson, I. C. M. and Nilsson, L. (2014). Semi-automated versus highly automated driving in critical situations caused by automation failures. Transportation Research Part F: Traffic Psychology and Behaviour, 27(B), 218-228.

80. Sun, Y., Olaru, D., Smith, B., Greaves, S. and Collins, A. (2017). Road to autonomous vehicles in Australia: an exploratory literature review. Road and Transport Research, 26(1), 34-47.

81. Talebpour, A. and Mahmassani, H. S. (2016). Influence of connected and autonomous vehicles on traffic flow stability and throughput. Transportation Research Part C: Emerging Technologies, 71, 143-163.

82. Thomopoulos, N. and Givoni, M. (2015). The autonomous car - a blessing or a curse for the future of low carbon mobility? An exploration of likely vs. desirable outcomes. European Journal of Futures Research, 3(1), 14.

83. Tinnilä, M. and Kallio, J. (2015). Impact of future trends on personal mobility services. International Journal of Automotive Technology and Management, 15(4), 401-417.

84. UK Government (2017). Press Release: Government Sets Out Next Steps in Establishing the $U K$ as Global Leader in Connected and Autonomous Vehicle. Available online: https://www.gov.uk/government/news/government-sets-out-next-steps-in-establishing-the-ukas-global-leader-in-connected-and-autonomous-vehicles (accessed 10 May 2018).

85. Victor, T., Rothoff, M., Coelingh, E., Ödblom, A. and Burgdorf, K. (2017). When Autonomous Vehicles Are Introduced on a Larger Scale in the Road Transport System: The Drive Me Project. In Automated Driving, 541-546. Springer, Cham.

86. Villagra, J., Milanés, V., Pérez, J. and Godoy, J. (2012). Smooth path and speed planning for an automated public transport vehicle. Robotics and Autonomous Systems, 60(2), 252-265.

87. von Ungern-Sternberg, A. (2018). Autonomous Driving: Regulatory Challenges Raised by Artificial Decision-Making and Tragic Choices. In: Woodrow Barfield and Ugo Pagallo (Eds), Research Handbook on the Law of Artificial Intelligence, Edward Elgar.

88. Waldrop, M. M. (2015). No drivers required. Nature, 518(7537), 20.

89. Wang, R., Li, Y. and Work, D. B. (2017). Comparing traffic state estimators for mixed human and automated traffic flows. Transportation Research Part C: Emerging Technologies, 78, 95110.

90. Wells, P. (2010). Sustainability and diversity in the global automotive industry. International Journal of Automotive Technology and Management, 10(2-3), 305-320. 
